\title{
Relevancia de las mipyme en el área metropolitana de Bucaramanga
}

Relevance of SMEs in the metropolitan area of Bucaramanga

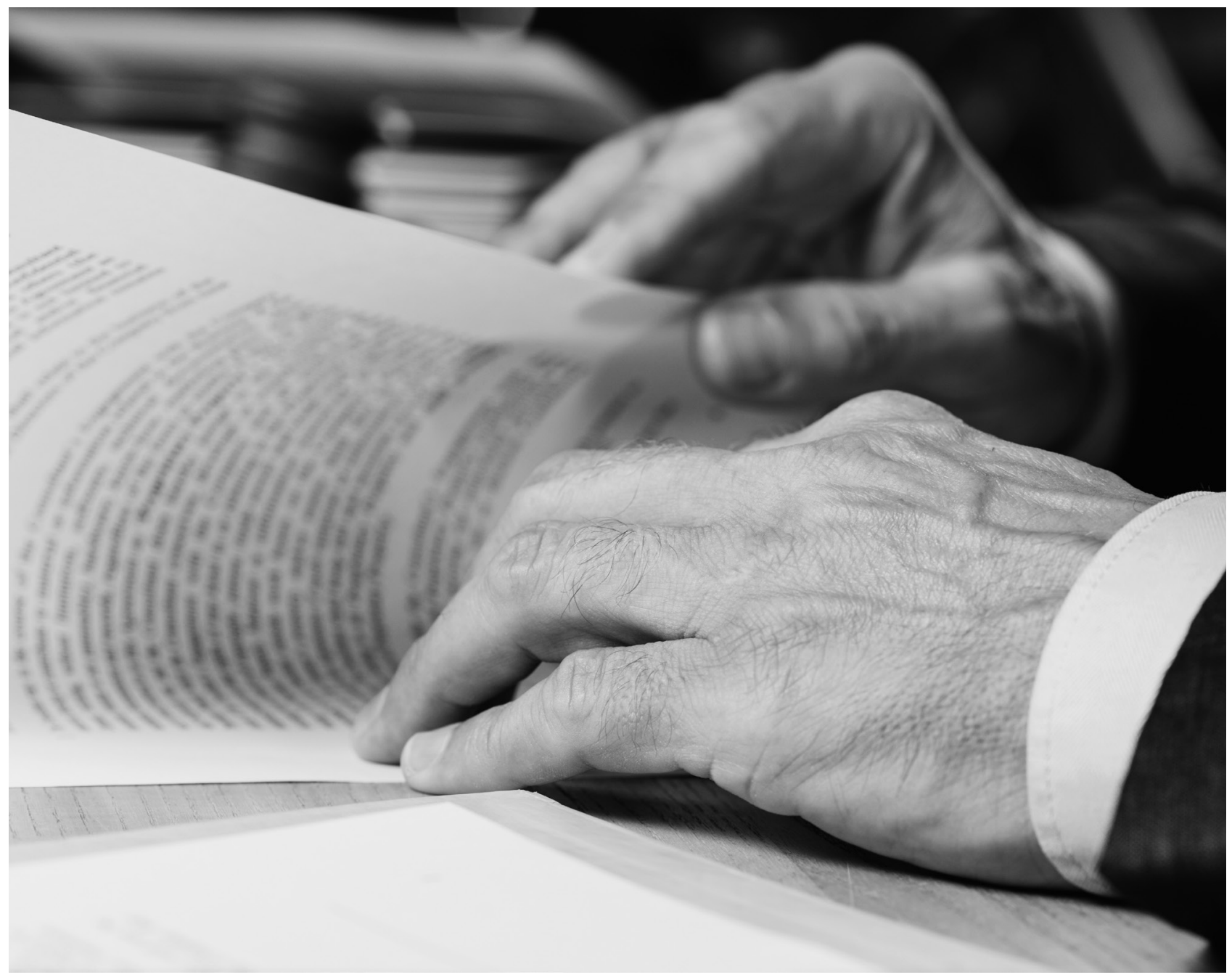




\title{
Relevancia de las mipyme en el área metropolitana de Bucaramanga ${ }^{1}$
}

\section{Relevance of SMEs in the metropolitan area of Bucaramanga}

\section{Andrés Leaño}

Artículo recibido en noviembre de 2017; artículo aceptado en mayo de 2018.

Este artículo puede compartirse bajo la Licencia Creative Commons Atribución-No Comercial-Compartir Igual 2.0 Genérica y se referencia usando el siguiente formato: Leaño, A. (2018). Relevancia de las Mipymes en el área metropolitana de Bucaramanga. I+D Revista de Investigaciones, 12 (2), 77-84. DOI: https://doi.org/10.33304/revinv.v12n2-2018008

\begin{abstract}
Resumen
Existe consenso en que las mipyme ejercen una gran influencia en la economía del área metropolitana de Bucaramanga, la investigación buscó contestar la pregunta acerca de cómo las empresas inciden en temas económicos como generación de empleo y crecimiento en sectores importantes para la metrópoli. El documento utiliza una metodología que combina literatura junto con las entrevistas y encuestas del trabajo de campo sobre las mipyme. Este proceso tiene como objetivo evaluar el acceso al impacto de las mipyme en la economía en lo que se refiere a la diversificación económica y la dependencia del país respecto del sector primario (minero-energético). Este estudio proporciona evidencia colaboradora de que las microempresas desempeñan un rol esencial en la economía diversificada.
\end{abstract}

Palabras clave: Mipyme, economía, crecimiento, área metropolitana, empresa.

\begin{abstract}
There is approval that the MSMEs exert a great influence in the economy of the metropolitan area of Bucaramanga. The research sought to answer the question of how companies influence economic issues such as job creation and growth in sectors important to the metropolis. The document uses a methodology that crosses the reader through a combination of literature along with interviews and fieldwork surveys on MSMEs. This process aims to access the impact of MSMEs on the economy in terms of economic diversification and country dependence on the primary sector (mining and energy). This study provides supporting evidence that microenterprises play an essential role in economic diversification economy.
\end{abstract}

Keywords: SMEs, economy, growth, metropolitan area, company.

\footnotetext{
1. El presente artículo es un avance de la investigación titulada "Las Mipymes en el marco del desarrollo local de Bucaramanga y su Área Metropolitana", que hace parte de la tesis de Maestría en Políticas de desarrollo (PolDes) de la Universidad Nacional de la Plata (UNLP) Argentina.

2. Andrés Leaño. Docente e investigador UIS, Trabajador social egresado de la Universidad Industrial de Santander (Colombia), Especialista en Pedagogía para el Aprendizaje Autónomo de la Universidad Nacional Abierta a Distancia (Colombia), Magíster en Políticas de Desarrollo en la Universidad Nacional de la Plata (Argentina), Magíster en Intervención social y comunitaria SIAC (España), ORCID ID: http://orcid.org/orcid. org/0000-0002-3736-0398. Correo electrónico: jandres1386@gmail.com.
} 


\section{Introducción}

El presente artículo es un apartado de la investigación referida al tema de las estrategias de crecimiento en las mipyme planteadas en los planes de desarrollo municipal [PDM] y su modelo de planificación del Área Metropolitana de Bucaramanga [AMB], Colombia, durante el periodo 2016 - 2020.

El área metropolitana de Bucaramanga está ubicada en la cordillera oriental de Colombia y es la tercera área metropolitana más importante del país, configurada por la Ley 128 de 1994. El AMB está conformado por Bucaramanga (centro y capital del Departamento de Santander) y los municipios aledaños de Floridablanca, Girón y Piedecuesta (periferia), y concentra buena parte de la industria manufacturera de la región; es una entidad autónoma administrativa con personería jurídica de derecho público, cuenta con patrimonio propio, autoridad y régimen fiscal especial.

Generalmente, se define como mipyme a las micro, pequeñas y medianas empresas, utilizando criterios como el volumen de ventas y el número de empleados en la empresa para evaluar el tamaño de estas. En Colombia, la Ley 590 de 2000, define las mipyme como micro, pequeña y mediana empresa, toda unidad de explotación económica realizada por persona natural o jurídica, en actividades empresariales, agropecuarias, industriales, comerciales o de servicios, rural o urbana. Dicha ley también describe sus características y las regula. Existen variados criterios que son asumidos por cada país para clasificar las mipyme; los criterios son de corte cuantitativo y cualitativo. Los criterios cualitativos representan el tipo de inserción en el mercado y la forma de administrar; las variables más utilizadas son:

- Acceso a innovación tecnológica y mercados de capital.

- Grado de especialización del trabajo y división especializada del trabajo.

- Establecimiento de las relaciones horizontales.

- La forma y el grado de competencia.

Por su parte, los criterios cuantitativos representan la información económica; la más utilizada por los países es la de ingresos, índice distorsionado porque no refleja las capacidades tecnológicas ni el mercado de la empresa (Argüello, 2015).

La Ley 1450 de 2011 utiliza la cantidad de empleados y sus ingresos para clasificarlas; vemos que se utilizan únicamente variables cuantitativas por la facilidad de medirlas. La definición y clasificación dada por la Ley 1450 de 2011, Artículo 2, Definiciones de tamaño empresarial, para todos los efectos, entiende por empresa toda unidad de explotación económica realizada por persona natural o jurídica, en actividades empresariales, agropecuarias, industriales, comerciales o de servicio, rural o urbano. Para la clasificación por tamaño empresarial, entiéndase micro, pequeña, mediana y gran empresa, se podrán utilizar uno o varios de los siguientes parámetros:

1. Número de trabajadores totales

2. Valor de ventas brutas anuales

3. Valor de activos totales

La Ley 590 de 2000 (ver Tabla 1), clasifica las mipymes así:

\section{Tabla 1 \\ Clasificación de las mipymes en Colombia}

Fuente: Autor

Un tercer criterio para la clasificación, que viene siendo estudiado en varios países, emplea criterios cuantitativos y cualitativos cruzando indicadores económicos, características de las políticas, actividades realizadas, estructura económica de la región y relación entre ingresos, etc.

Por otro lado, los emprendimientos empresariales, según Hodgetts y Kuratko (2002), son aquellos cuyos objetivos principales son la rentabilidad y el crecimiento. Richard Daft (2000) y muchos otros autores definen el emprendimiento, de manera tradicional, como el proceso de iniciar una empresa, organizar los recursos necesarios y asumir el riesgo y la recompensa asociados.

\section{Impacto de las mipyme en la economía metropolitana}

Para la creación de empleos es importante darse cuenta de que las microempresas juegan un papel importante en la economía al proporcionar empleo como bienes y servicios; además, se incluye a las pequeñas o medianas empresas haciendo lo que ellas no pueden o no quieren 
hacer. Diversos autores (Gómez \& Sarmiento, 2011; Hallberg, 2000; Harris, 2000; Lundvall, 1992; Medina, Patiño \& Rodríguez, 2011; Montenegro \& Ramírez, 2006; Naclerio, 2010; Nelson, 1993), señalan que las microempresas crean un gran porcentaje de nuevos puestos de trabajo. Las micro tienden a ser más intensivas en trabajo que intensivas en capital. Los empleos creados por estas empresas dan a la metrópoli una ventaja económica sobre otras ciudades, especialmente en el sector manufacturero (Krugman, 1997). En cualquier economía los empleos se están creando a través de los nacimientos y la expansión de las microempresas; a la inversa, los empleos se están perdiendo mediante la reducción de personal y el cierre de estas pequeñas empresas. El efecto neto de estos nacimientos y muertes puede ser algo volátil en el mercado de trabajo (Gómez, 2014).

De acuerdo con Hallberg (2000), aunque las tasas de creación de empleo en las micro son significativamente más altas que en las empresas más grandes, también lo son sus tasas brutas de destrucción de puestos de trabajo. Así, las microempresas experimentan altas tasas de natalidad y de mortalidad e incluso pueden no crecer. Por lo tanto, estas empresas pueden ofrecer menos seguridad en el empleo que sus contrapartes pequeñas y medianas.

Sin embargo, parece que la destrucción de puestos de trabajo durante la recesión es menor para las microempresas que en las otras empresas. Los propietarios de las micro, en comparación con las pequeñas o medianas empresas, están más dispuestos a aceptar reducciones de remuneraciones y retornos durante una recesión para evitar el cierre. Por lo tanto, parecen tener mayor flexibilidad salarial.

\section{Tipologías de empleo}

Las microempresas tienen más probabilidades de emplear a trabajadores menos calificados y a personas sin experiencia laboral previa. Esta práctica de empleo beneficia a la economía de dos maneras.

En primer lugar, las micro emplean a trabajadores que de otro modo podrían tener dificultades para conseguir empleos, reduciendo así la duración del desempleo transitorio. En segundo lugar, el empleo de estos trabajadores aumenta su productividad, dándoles capacitación en el trabajo y experiencia laboral.

\section{Productividad}

Las microempresas usualmente tienen una propiedad menos difundida, una estructura organizativa menos compleja y es más probable que el propietario participe directamente en la gestión. Como resultado, los propietarios y los gerentes pueden observar y asegurar con más facilidad la productividad de los empleados; por el contrario, las micro tienen una productividad más baja que las mayores, como se refleja en los salarios comparativamente más bajos y los beneficios adicionales ofrecidos por los propietarios de las micro a sus empleados respecto de las empresas de mayor tamaño. Además, estas empresas tienen menos probabilidades de experimentar los beneficios de ahorros en costos asociados con la producción en una gran venta frente a las economías de escala.

\section{Disminución del desempleo}

Las empresas juegan un rol fundamental en la reducción del desempleo y el gobierno nacional o local crea programas para fomentar la creación de empresas mediante subsidios al desarrollo de microempresas con préstamos con intereses bajos y subvenciones, lo que puede causar un efecto de peso muerto y de desplazamiento. El primero se refiere al costo para la sociedad de otorgar fondos públicos a empresas que, en cualquier caso, emprenderán esa actividad. El efecto de desplazamiento ocurre cuando la nueva pequeña empresa subvencionada compite y desplaza a las entidades existentes no subvencionadas.

\section{Inflación}

Las microempresas tienen una mayor flexibilidad que las pequeñas o medianas empresas al ajustar sus niveles de producción y, por lo tanto, son capaces de acomodar fluctuaciones aleatorias a corto plazo en la demanda. Al actuar como amortiguadores para demandar los choques, las microempresas ayudan a satisfacer aumentos temporales de la demanda sin provocar aumentos bruscos de precios. Por lo tanto, proporcionan mayor competencia y flexibilidad a través de los sectores, ayudando a mantener presiones inflacionarias.

Por lo tanto, las mipyme desempeñan un papel importante en la reducción de la inflación, dado que las presiones inflacionistas sostenidas pueden neutralizar los beneficios económicos obtenidos a través de la generación de empleo y el crecimiento económico.

\section{El trabajo de campo}

El AMB posee una población de 1122945 habitantes, cuenta con 62050 microempresas, 2798 pequeñas empresas y 683 medianas empresas para un total de 65531 mipyme registradas ante la Cámara de Comercio de Bucaramanga entre 2014 y 2015. 
El Área Metropolitana es reconocida por ser una metrópoli de bajo desempleo, en especial, el centro de Bucaramanga, que ofrece altas tasas de cobertura educativa y cuenta con población laboriosa y emprendedora; sin embargo, presenta altos porcentajes de informalidad y subempleo, y sus pobladores buscan autoemplearse mediante un proyecto empresarial. La actividad productiva industrial del municipio con mayor posicionamiento es la marroquinería y el calzado, cuyos productos se exportan a países como Argentina, Brasil, Canadá, Ecuador, Estados Unidos, Venezuela y países de la Alianza del Pacífico; además, se destacaban las actividades de confección y orfebrería. Otro renglón importante es la prestación de servicios, como salud, educación y finanzas.

Para analizar el problema de la informalidad y el subempleo es necesario de mencionar sus causas; si bien la metrópoli pertenece a una región periférica como es Latinoamérica, sabemos que la estructura productiva es desequilibrada, razón por la cual las empresas e industrias son incapaces de recibir la mano de obra ofertada.

Para caracterizar la estrategia central se emplearon la encuesta y la entrevista a los empresarios que tenían registrada su unidad productiva ante la Cámara de Comercio de Bucaramanga. Otras fuentes de información utilizadas fueron el Observatorio Metropolitano de Bucaramanga, la Cámara de Comercio de Bucaramanga [CCB] y el Departamento Administrativo Nacional de Estadística [DANE].

Laencuesta es un procedimiento para obtenerinformacióny se utiliza para recopilar una amplia muestra de encuestados. En el caso de esta investigación, se utilizaron encuestas electrónicas y personales; al mismo tiempo se realizaron las entrevistas semiestructuradas, caracterizadas por su flexibilidad y preguntas abiertas donde el entrevistador lleva una guía con los temas a preguntar. Las encuestas y las entrevistas fueron los instrumentos aplicados a 32 empresarios registrados ante la CCB en los meses de enero a marzo del 2016; el cuestionario está estructurado en 9 secciones, con 42 preguntas.

El cuestionario fue aplicado a manera de prueba en 5 mipyme para la retroalimentación y corrección de fallas; se hicieron ajustes menores para mayor confiabilidad; la aplicación y recolección fue realizada por el autor de la investigación. Al mismo tiempo, se aplicó la entrevista con preguntas semiestructuradas como complemento a la encuesta, lo que permitió mayor grado de profundidad en el conocimiento del objeto de estudio.

El AMB tiene una marcada vocación empresarial que representa el $40 \%$ del PBI departamental y según el Observatorio Metropolitano de Bucaramanga existen 65531 empresas activas a diciembre del 2015, de las cuales el $94 \%$ son microempresas, $4 \%$ pequeñas y el $1 \%$ medianas empresas, lo que significa una alta concentración de microempresas menores de 10 empleados y de baja o nula complejidad.

Las actividades económicas preponderantes en el AMB son el comercio, los restaurantes, los hoteles y otros, con el $51 \%$ de la actividad económica. Están divididas en $80 \%$ ventas de comercio al por mayor y menor, $16 \%$ alojamiento y $4 \%$ otros, lo que permite observar la baja complejidad de la mayor actividad económica de la metrópoli.

\section{Diagnóstico de las mipyme}

Un total de 32 encuestas y entrevistas realizadas permitió explorar las mipyme e identificar variables y comportamientos de los empresarios.

Se tomó el 0,05\% por municipio, es decir, la participación por municipios fue de 21 encuestas para Bucaramanga, 6 para Floridablanca, 3 para Girón y 2 para Piedecuesta.

\section{Tabla 2}

\section{Participación de municipios}

\begin{tabular}{cc} 
Porcentaje & Municipios \\
\hline $66 \%$ & Bucaramanga \\
$19 \%$ & Floridablanca \\
$91 \%$ & Girón \\
$6 \%$ & Piedecuesta \\
\hline $100 \%$ & Total general
\end{tabular}

Fuente: Autor

El $97 \%$ de las encuestas corresponden a microempresas, es decir, que no superan los 10 trabajadores o tienen activos inferiores a 501 SMMLV; el restante $3 \%$ se refiere a pequeñas empresas, es decir, aquellas donde trabajan entre 11 y 50 personas o poseen con activos entre 501 SMLV y 5001 SMMLV.

\section{Tabla 3 \\ Tamaño de la empresa}

\begin{tabular}{cc} 
Tamaño de la empresa \\
\hline $97 \%$ & Microempresa \\
$3 \%$ & Pequeña \\
\hline $100 \%$ & Total general
\end{tabular}

Fuente: Autor 
Como se observa en las tablas 2 y 3, hay una alta concentración de microempresas (97\%), en especial en el municipio de Bucaramanga con el $66 \%$.

\section{Género}

femenino Masculino

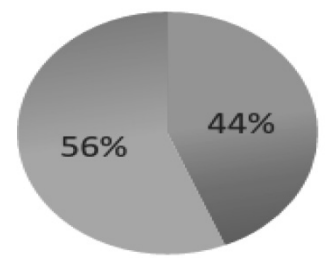

Figura 1. Género. Fuente: Autor

Por su parte, en cuanto a la distribución de la variable demográfica género entre los empresarios, para el caso del $A M B$, se presenta una mayor concentración del género masculino, con $56 \%$, frente al $44 \%$ femenino; esta diferencia del $12 \%$ indicaría una relativa igualdad de género en temas empresariales en la metrópoli.

A pesar de los datos, una empresaria en la entrevista manifestó que "hay hombres que les da envidia que uno sola les gane, que culpa que yo venda más" (Leaño, 2016), lo que dejaría entrever la rivalidad de género que existe en los empresarios del AMB.

\section{Sector económico}

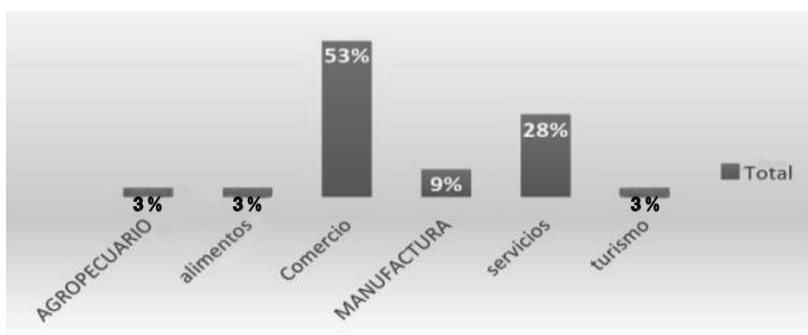

Figura 2. Sector económico. Fuente: Autor

Según los resultados obtenidos, el sector predominante es el comercial, con un $53 \%$, seguido por servicios con el $28 \%$. Asimismo, se encontró una baja participación de la industria y otros sectores que durante mucho tiempo habían sido fuertes, lo que denota un proceso de transición productiva de sectores agropecuarios e industria manufacturera al sector comercio y servicio.

Esta transición productiva deteriora la industria, la cual le añade valor agregado a los productos típicos de la región, fundamental en el desarrollo local de la metrópoli.
Para el caso de Bucaramanga, el mayor porcentaje del rango de edad de los empresarios intervenidos oscila entre 18 a 25 años, con un porcentaje del $25 \%$, seguido de 26 a 33 años con el $21 \%$, rangos de edad con disposición para el trabajo, con edades intermedias de experiencia y conocimiento, con marcada flexibilidad para la apertura y la innovación. Esto indica que en las empresas los diferentes rangos de edad están involucrados y convergen entre sí.

El sector de los procesadores de alimentos y el subsector de bebidas tienen un peso mediano frente al sector predominante, pero a pesar de ello contribuyen al empleo; este resultado puede explicarse tanto por el bajo número de mipyme en este sector como por la gran demanda que estas industrias hacen de mano de obra. Por su parte, las industrias de productos químicos pesados tecnológicos y minerales no metálicos contribuyen menos a la generación de empleo en la industria manufacturera, así como los subsectores declinantes de textiles, prendas de vestir, calzado y sombreros.

La contribución al empleo total por parte de las mipyme al sector manufacturero aumentó de 5840 en 2000 a 7152 en 2006, lo que representa un aumento del $18 \%$ durante el período. Este aumento ocurrió en contra de la disminución del número total de empleados en la manufactura, que pasó de 54800 en 2002 a 58400 en 2006. Este resultado hace hincapié en el impacto positivo que tienen las mipyme en el sector manufacturero de la economía en términos de generación de empleo, mientras que otras empresas más grandes del sector parecen estar exigiendo menos trabajadores. Sin embargo, la reducción de empleos no implica necesariamente una disminución o una menor eficiencia / productividad de estas empresas más grandes, ya que pueden estar sustituyendo más capital por mano de obra, especialmente a la luz de las presiones de globalización aceleradas por la liberalización del comercio.

En lo que respecta al sector de los servicios, es interesante destacar la posición dominante del sector de la distribución como uno de los principales contribuyentes a la generación de empleo tanto en las grandes empresas como en las micro.

\section{PIB y valor agregado}

El concepto de valor agregado es muy importante para calcular el PIB a través del método de producción, debido a que evita el doble conteo. Este método no se limita a considerar la producción absoluta, sino que registra el cambio en la producción ocasionado por los insumos de capital, trabajo y tierra en cada etapa de la producción. 
Se espera que las mipyme contribuyan significativamente al PIB debido, principalmente, a su gran número y a los efectos de generación de ingresos en forma de salarios y salarios pagados a los trabajadores, así como los beneficios dirigidos a los empresarios. Por lo tanto, las mipyme desempeñan un papel vital en el servicio de los mercados localizados. Además, con referencia a los bienes intermedios/valor agregado, muchas microempresas existen para atender demandas particulares, como en el caso de bienes de consumo para los cuales existe una diversidad considerable de gustos; en particular, las microempresas son importantes proveedores de bienes intermedios especializados, es decir, bienes utilizados en la producción de otros bienes. Así, de gran importancia son los efectos de la mipyme sobre el sector manufacturero en lo que respecta a las contribuciones del PIB.

En cuanto a los propietarios de las empresas en el sector manufacturero, su impacto total sobre el PIB puede verse limitado por la falta de innovación. En efecto, (Narodowski, 2007) señaló que "(...) la tecnología, para la clase industrial local, se supone que es un bien extranjero, exógeno a la sociedad. La innovación en la tecnología como ciencia aplicada a la producción no forma parte de la cultura local. Práctica estándar para los fabricantes es elegir un producto para el cual la demanda es significativa y luego proceder a acceder a la tecnología a través de la compra, o por la asociación con algún agente extranjero". Por lo tanto, el valor añadido total / potencial del sector manufacturero no está siendo alcanzado tanto por las micro como por las pequeñas o medianas empresas.

\section{Diversificación económica}

El espíritu empresarial ofrece oportunidades a las personas que pueden sentirse excluidas del entramado productivo. Por lo tanto, las microempresas desempeñan un papel importante en la inclusión y la diversificación económica, introduciendo nuevos bienes y servicios. (Manual de Oslo, 2005).

La Figura 2 revela que la mayoría de las mipyme contribuyen en gran medida a la diversificación de la economía, como puede verse a través de su distribución en los subsectores que tienen una concentración marginalmente más elevada.

Al estudiar la manufactura y los servicios, parece que hay grandes oportunidades para establecer vínculos. Según Gómez y Sarmiento (2011) durante el período 1995 -1999 el mercado local estaba fuertemente protegido. Esto estimuló una mayor competencia entre las pequeñas empresas para mantener o aumentar su cuota de mercado, resultando así en poco o ningún vínculo entre ellas. Sin embargo, desde el inicio de los años ochenta presenciamos la apertura del mercado a la competencia extranjera como consecuencia del ajuste estructural. Como resultado, las pequeñas empresas encontraron que era necesario formar mayores vínculos y se reformularon para centrarse en el ahora liberalizado mercado de exportación.

La apertura comercial en el país a mediados de la década del noventa generó oportunidades y amenazas para las empresas locales. Por lo tanto, en el entorno globalizado y liberalizado existente, estas mipyme deben ser capaces de medirse con competidores extranjeros en el país (multinacionales) y en el extranjero. Según Naclerio, (2010): “En un ambiente de ritmo rápido por la competencia extranjera, los empresarios locales tienen que ser dinámicos e innovadores para sobrevivir, ya sea a nivel de microempresas, pequeñas o grandes empresas". Por lo tanto, la realidad de la competencia debe tener el potencial de despertar a los microempresarios para aumentar sus niveles de creatividad y riesgos. Esos esfuerzos son importantes para agregar mayores oportunidades de vinculación y diversificación de la economía a través de productos nuevos (Malthus, 1970). El impacto económico puede disiparse ya que muchas grandes empresas multinacionales tienen estrechos vínculos con su empresa matriz y poco o ningún comercio con las manufacturas locales. De hecho, la proliferación de franquicias extranjeras de comida rápida obliga a los administradores de estos establecimientos a comprar insumos y accesorios extranjeros que podrían haber sido suministrados por microempresas.

\section{Conclusiones}

Hemos visto que las mipyme tienen un impacto importante en la economía del área metropolitana de Bucaramanga; son claros sus efectos positivos sobre la generación de empleo en el subsector, el crecimiento económico, el establecimiento de vínculos, la diversificación de la economía y los controles inflacionarios.

Hay que destacar la expansión de las mipyme como motor para el crecimiento de la metrópoli, favoreciendo el progreso del bienestar, proporcionando caminos hacia la reducción sostenible de la pobreza, el alivio del desempleo, la mejora de la comunidad y el empoderamiento general de los grupos desfavorecidos.

\section{Referencias}

Argüello, G. M. (2015). Uso de las TIC en las PYME del sector industrial del Área Metropolitana de Bucaramanga. 
1+D Revista De Investigaciones, 6(2), 125-136. Recuperado de https://doi.org/10.33304/revinv.v06n2-2015009

Congreso de Colombia. (2000). Ley 590 del 2000. Publicado en Diario Oficial de la República de Colombia, No. 44.078 de 12 de julio 2000. Bogotá.

Congreso de Colombia. (2011). Ley 1450 del 2011. Diario Oficial de la República de Colombia, No. 48.102 del 16 de julio 2011. Bogotá.

Daft, R. (2000). Teoría y diseño organizacional (6a. ed.). México: International Thomson.

Gómez, F. S. (2014). Colombia en la inserción de la economía internacional. $1+D$ Revista De Investigaciones, 4(2), 104-111. Recuperado de https://doi.org/10.33304/revinv.v04n2-2014009

Gómez, E. \& Sarmiento, G. (2011). Proceso de internacionalización de empresas del área metropolitana de Bucaramanga. Revista Lebret, 3.

Hallberg, K. (2000). Una estrategia orientada al mercado para las pequeñas y medianas empresas. (Corporación Financiera Internacional, Documento de Discusión No. 40). Washington, D. C.: Banco Mundial.

Harris, M. (2000). Situación actual de las pequeñas y medianas empresas industriales en Trinidad y Tobago, Barbados y Santa Lucía. Desarrollo Productivo, 69. Santiago, Chile: Naciones Unidas.

Hodgetts, M. \& Kuratko, F. (2002). Gestión eficaz de la pequeña empresa. Hinsdale, Illinois: Dryden Press.

Krugman, P. (1997). Desarrollo, geografía y teoría económica. Barcelona: Antony Bosch.

Leaño, A. (2016). Las Mipymes en el marco del desarrollo local de Bucaramanga y su Área Metropolitana: Universidad Nacional de La Plata. Recuperado de http://www.memoria.fahce.unlp.edu.ar/tesis/ te.1436/te.1436.pdf

Lundvall, B. (1992). National systems of innovation: Towards a theory of innovation and interactive learning. London: Pinter Publishers.

Malthus, R. (1970). Primer ensayo sobre la población. Madrid: Alianza Editorial.

Manual de Oslo, (2005). Directrices para la recolección e interpretación de datos sobre innovación (3a. ed.). México: OCDE / Finep.

Montenegro, C. \& Ramírez, H. (2006). Estudio prospectivo de las mipymes del subsector calzado de Bucaramanga y su área metropolitana (Tesis de pregrado). Bucaramanga: Universidad Industrial de Santander.

Naclerio, A. (2010). Sistemas productivos locales, políticas públicas y desarrollo económico: Programa Naciones Unidas Para el Desarrollo.

Narodowski, P. (2007). La Argentina pasiva: Desarrollo, subjetividad, instituciones, más allá de la modernidad: el desarrollo visto desde el margen de una periferia, de un país dependiente. Buenos Aires: Prometeo Libros.

Nelson, R. (1993). National Innovation Systems: A
Comparative Analysis: Oxford University Press. 